\title{
Squamous Cell Carcinoma Arising in a Dermoid Cyst of the Ovary: A Case Series
}

\author{
Hossain $\mathrm{N}^{1^{*}}$, Nag $\mathrm{U}^{1}$, Mahmud MS ${ }^{2}$ \\ ${ }^{I}$ Department of Gynecological Oncology, National Institute of Cancer Research \& Hospital, Dhaka, \\ Bangladesh; ${ }^{2}$ Combined Military Hospital, Dhaka, Bangladesh
}

\begin{abstract}
Background: Malignant transformation in a dermoid cyst of the ovary is a rare complication, occurring in only $1-2 \%$ of cases, with squamous cell carcinoma being the most common type. Preoperative diagnosis is difficult because of the lack of specific symptoms and signs to suggest malignancy. The prognosis is generally poor when disease has spread beyond the ovary.
\end{abstract}

Objective: This study was conducted to review experience with this disease and describe the current treatment modality.

Methods: It was identified 4 women with this diagnosis during 2013-2018. This is a descriptive study, looking at the patient's characteristics, mode of presentation, the role of tumor markers and radiological imaging in diagnosis. It was also examined the stage and pathological features of the presentation and subsequent course of the disease.

Result: The median age was 45 (range: $32-63$ yrs). Three cases were stage I and one case was stage III. The average tumor diameter was $14.1 \mathrm{~cm}$. All patients were under surgery. Two patients not received any adjuvant therapy and two patients received adjuvant chemotherapy. One patient had recurrent disease in the pelvis during adjuvant chemotherapy and died at 8 month of her diagnosis. Other three patients were disease free for last 3 years. The overall 2 yrs survival was $70 \%$.

Conclusion: Squamous carcinomas arising in mature cystic teratomas are commonly large ovarian tumors that occur in perimenopausal women often present as an incidental finding. There are no reliable diagnostic tools or prognostic indicators. The behaviour of these tumors is unpredictable. Conservative surgery may be considered in women wishing to preserve fertility. The role of chemotherapy and radiotherapy remains unclear in the adjuvant or metastatic setting.

\section{Introduction}

Dermoid cyst or mature cystic teratoma (MCT) is the most common germ cell tumor of the ovary and accounts for $10-20 \%$ of all ovarian tumors. ${ }^{1}$ Malignant transformation occurs in $1-2 \%$ of MCT and majority of these $(80-90 \%)$ are squamous cell carcinoma (SCC) followed by adenocarcinoma.,

Squamous cell carcinoma (SSC) in MCT is most commonly seen in postmenopausal women. ${ }^{4}$ Clinical presentation is similar to other ovarian tumors, tumor markers are not often raised and imaging methods are many times not helpful. Hence, most cases are diagnosed postoperatively. ${ }^{5}$

Tumor confined to the ovary usually have a better prognosis and patients with stage III or IV disease rarely survive five years. ${ }^{5}$ Because of the relative rarity of these tumors, our knowledge about this tumor type is limited. The literature is limited to descriptive case series and there is no consensus regarding optimal management.

This study was aimed to further characterize the population of women affected by this disease, the

*Correspondence: Nasrin Hossain, Department of Gynecological Oncology, National Institute of Cancer Research \& Hospital, Dhaka, Bangladesh; e-mail: nasrinhossain23@gmail.com; ORCID: https://orcid.org/0000-0002-0467-434X disease itself and the most appropriate course of management.

Cases: It was identified 4 women diagnosed with squamous cell carcinoma arising in a dermoid cyst of the ovary between 2014-2018. All the pathologic diagnoses were confirmed in our pathology department (NICRH pathology department) and a retrospective review of the patient's case notes was carried out.

Case 1:A 63 years old menopausal lady,para-2, presented with chronic symptoms with abdominal pain, urinary symptoms and anorexia/weight loss. There was no particular past gynecological, medical or family history. Per-abdominal exam showed a large firm to hard, non-tender, welldefined, mobile mass in lower abdomen. Pelvic examination revealed a mobile mass on right side moving with cervical movement. Routine and specific investigation were carried out. USG whole abdomen suggested the possibility of a dermoid cyst of the ovary (size about 16*12) (side not specified). Tumour markers were normal. Laparotomy was done-TAH + BLSO + omental 
sampling + whole abdominal exploration. It was stage IA. HPR revealed-Squamous cell carcinoma in dermoid cyst. Then she received 6 cycle chemotherapy (pac+carbo). She was completely well for 36 months since then.

Case 2: Mrs. Tahmina, 32 years, a regularly menstruating lady, para-1 presented with acute abdomen. Through physical examination and investigation diagnosed a case of twisted ovarian tumor probably due to dermoid cyst. Laparotomy followed by right sided unilateral salpingoophorectomy was done and incompletely staged. Histopathology report revealed - squamous cell carcinoma in the dermoid cyst. Then whole abdomen CT scan and tumor marker CA125 was done and found normal. Patient was placed in tumor board; according to tumor board decision, it was only follow up, no further surgery or adjuvant therapy. Patient was remained well with no evidence recurrent disease for 24 months since then.

Case 3: Mrs. Niva, 37 years, para-2, and a regularly menstruating lady presented with abdominal lump, abdominal pain. Per abdominal examination showed a large firm to hard, slightly tender, well defined mass in the lower abdomen. Pelvic examination revealed a large mobile mass felt through right fornix. Routine and specific investigations were carried out. USG suggested a complex mass size about $10 \times 8 \mathrm{~cm}$ in the pelvis. Tumor markers were normal. Laparotomy followed by TAH with BLSO with surgical staging was done. HPR revealed: left ovarian tumor: SCC in dermoid cyst, Right ovarian tumor: Mature cystic tumor. Final staging was stage IA. A patient was placed in tumor board and decision was only follow up with any adjuvant chemotherapy/ radiotherapy. Patient was disease free for 30 month, up to date.

Case 4: Mrs Fulmoti, 48 years, para-4, presented with abdominal lump, pain, loss of weight. General condition was poor. Per abdominal examination showed a large, ill defined mass in the lower part of abdomen, slightly restricted mobility, ascites present. Pelvic exam revealed mass felt through the fornix. Per rectal exam revealed mass in the POD. USG of whole abdomen suggested the large (18*16) complex mass in the pelvis and ascites present. CT scan of whole abdomen revealed complex mass in the bladder and rectal wall. Ca125 tumor marker was raised. Staging laparotomy was done- TAH with BLSO with infra-colic omentectomy was done. HPR- SSC in the dermoid cyst in left side and omental metastasis was present, o tumor stage was IIIC. Then decided to adjuvant chemotherapy. She received 2 cycles CT (carbo+pac) and discontinues the chemotherapy due to toxicity. Again developed lump in the abdomen after 4 months. Patient was died at 8 months after her diagnosed.

\section{Discussion}

Squamous cell carcinoma (SCC) arising from a mature cystic teratoma (MCT) is a rare pathological event that is not diagnosed preoperatively. There are no particular signs and symptoms which are characteristic of malignancy arising in a dermoid cyst.

Due to its rarity, the surgical and postoperative treatment of this malignancy is not established in the literature. Since mature cystic tumor is a common ovarian neoplasm and it is increasingly diagnosed as an incidental finding in a wellimaged patients population. There has been growing emphasis on current opinion is that the women's age at presentation with malignant transformation in a dermoid cyst is older than those with benign disease and is common in postmenopausal women. ${ }^{3,4}$ The average age at presentation in our series was 45 years which would be in keeping with these previous report. ${ }^{1.2}$ There did not appear to be a correlation between age and prognosis in our women. Taka shina et al. ${ }^{6}$ demonstrated a role for patients age in differential diagnosis, with a mean age at diagnosis of 32.7 years for mature dermoid cyst versus $50.8 \mathrm{yrs}$ for SCC in dermoid cyst. So, it would be suggested that age is not necessarily a prognostic but may be useful in terms of raising suspicious of malignant transformation when an older women presents with what appears to be a benign dermoid cyst.

Tumor size had also been noted to predict malignancy. Although mature cystic teratoma presents in a wide range of sizes, larger tumors correlate with an increased risk of malignant transformation. In the current series, the average tumor diameter was $14.2 \mathrm{~cm}$, considerably larger than a typical benign cyst. It was to be found, in keeping with most other published series that preoperative imaging with USG was of little value in predicting this diagnosis. Several radiological studied have suggested tumor imaging characteristics that may be useful in preoperative risk assessment. Transvaginal colour doppler ultrasound and MRI may predict the preoperative risk assessment. ${ }^{1,5}$

Are tumor markers useful in term of preoperative diagnosis? In this series, CA125 was only documented as high in one of our four women and as normal in three women. This is imply a lack of 
usefulness as a diagnostic tool and would be in keeping. Current published data suggesting that CA125 does not predict this diagnosis preoperative..$^{2,7}$

In term of predicting likelihood of disease recurrence, stage appeared to be the most useful prognostic. Generally, women with Stage IA disease have an excellent prognosis (remaining disease free 3 years, 2 years and 2.6 years after diagnosis in our series) and one case, stage IIIC died 8 months of her disease. ${ }^{2}$

There is no clear consensus on an optimal management strategy for these women. Most centres would advocate that initial treatment should be with surgery and these were the case in all our women. TAH with BLSO with omentectomy would be the widely accepted approach, as this allows full staging of disease.

Optimal de-bulking if metastatic disease is present is also thought to improve survival in this case, as it does for primary epithelial tumors. One of these women (who was aged 35 yrs or younger)was initially treated with unilateral oophorectomy. Now, she is disease free at least 2 years from disease. The question as to whether fertility sparing surgery is appropriate for young women with disease localized to one ovary has never been definitively answered.

Due to the rarity of this tumor adjuvant treatment has not been prospectively evaluated. For young patients with intact stage IA disease and fertility consideration, conservative treatment with unilateral salpingo-oophorectomy and surgical staging and close follow-up has been proposed. ${ }^{2}$

In women deemed at high risk of disease recurrence, post operative radical pelvic radiotherapy may be considered. ${ }^{2}$ The rational for this is that in patients who died recurrence, pelvic disease was common and a particularly difficult problem to manage clinically but little benefit of radiotherapy in the metastatic setting. ${ }^{2}$

In term of chemotherapy, it would be fair to conclude from previous studies that chemotherapy is less effective in this disease than in primary epithelial ovarian tumors. ${ }^{7}$ While some case series have reported some evidence of chemo sensitivity. It was observed that no definitive benefit from chemotherapy in any of these women, either in the adjuvant or metastatic setting.

Most women in this series (in common with others reported $)^{4,5}$ had regimens used to treat epithelial ovarian cancer, it is possible that regimens more commonly used in the treatment of squamous tumors such as cisplatin and 5 flurouracil may have demonstrated more convincing evidence of activity. Concurrent chemo radiation has also been advocated by several authors in a similar manner to that used for squamous cell carcinoma of the cervix, with varying results. ${ }^{7}$

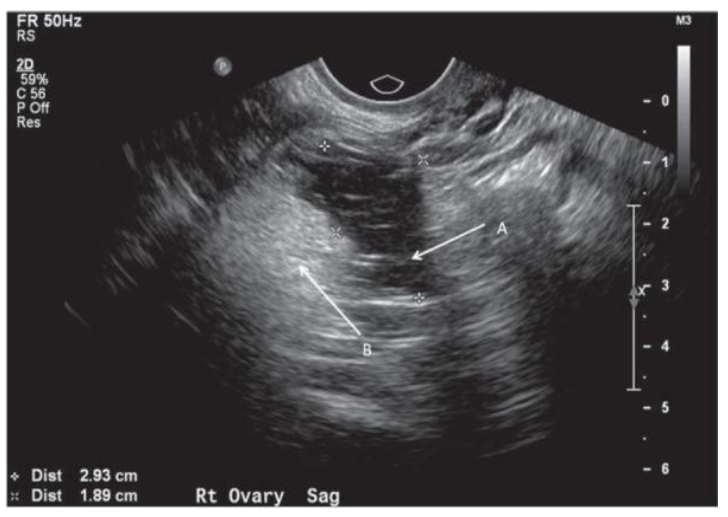

Figure 1: Ultrasonography of Dermoid cyst.

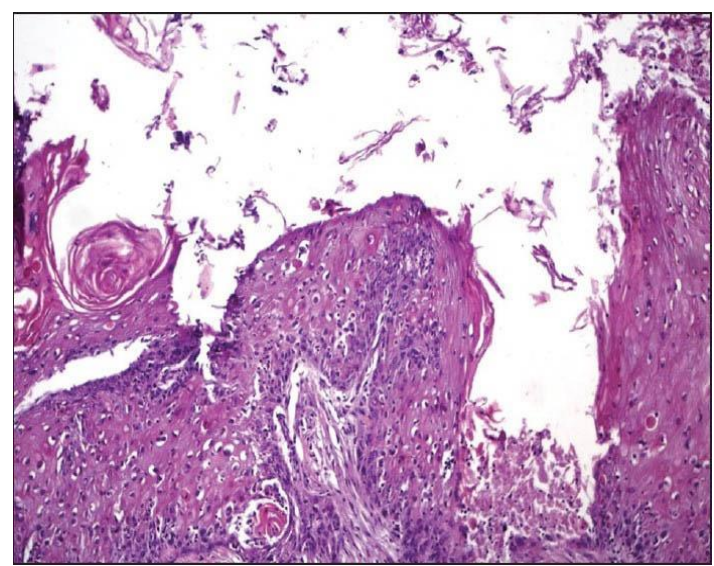

Figure 2: Histopathology of Squamous cell carcinoma in dermoid cyst

\section{Conclusion}

In conclusion, SCC arising in Mature Cystic Teratoma (MCT) presents a diagnostic and therapeutic dilemma. Based on the authors' experience with this disease and currently available data, we recommend vigilant preoperative risk assessment, surgical cytoreduction with proper staging, and adjuvant therapy with platinum-based and concurrent whole pelvic radiation in surgically confirmed earlystage disease. Patients with stage-III disease have a poor prognosis and should be offered platinumbased chemotherapy. Keeping in view the rarity, it is very essential to be aware of this condition and be equipped to deal with it. 


\section{References}

1. Lisa Dos, Evelyn, alexia et al. Squamous cell carcinoma arising in mature cystic teratoma of the ovary: A case series and review of the literature. Gynecologic Oncology. 2007;105:321-324. doi:10.1016/j.ygyno.2006.12.008

2. JL Hurwitz, A fenton, WG Mccluggage et al. Squamous cell carcinoma arising in a dermoid cyst of the ovary; a case series. BJOC. 2007;114:1283-1287. doi: 10.1111/j.1471-0528.2007.01478.x

3. Powell JL, Stinson, Cannor et al. Squamous cell carcinoma arise from dermoid cyst of the ovary. Gynecol Oncol. 2003:69; 526-8.
4. Tangjitgamol S, Manusirivithaya S, Sheanakul C et al. Squamous cell carcinoma arising from dermoid cyst: case reports and review of literature. Int J Gynecol Cancer. 2003; 13:558-63.

5. Ranu Patni. Squamous cell carcinoma arising in mature cystic teratomo of ovary. Journal of Midlife Health. 2014;5:195-97.

doi: 10.4103/0976-7800.145169.

6. Kashimura M, Shinohara M, Hirakawa $\mathrm{T}$ et al. Clinicopathologic study of squamous cell carcinoma of the ovary. Gynecol Oncol. 1989; 34: 75-9.

7. John L, Powell M.D, Jeffrey A et al. Gynecologic Oncology. 2003;89: 526-528. doi: 10.1016/s0090-8258:00135-5. 